(C) 1998 International Press

Adv. Theor. Math. Phys. 2 (1998) 119-153

\title{
Light-Cone Description of $(2,0)$ Superconformal Theories in Six Dimensions
}

\author{
Ofer Aharony ${ }^{a, 1}$, Micha Berkooz ${ }^{b, 2}$, Nathan Seiberg $^{b, 3}$ \\ ${ }^{a}$ Department of Physics and Astronomy \\ Rutgers University \\ Piscataway, NJ 08855-0849, USA \\ ${ }^{b}$ Institute for Advanced Study \\ Princeton, NJ 08540, USA
}

\begin{abstract}
We study the $(2,0)$ superconformal theories in six dimensions, which arise from the low-energy limit of $k$ coincident 5 -branes, using their discrete light-cone formulation as a superconformal quantum mechanical sigma model. We analyze the realization of the superconformal symmetry in the quantum mechanics, and the realization of primary operators. As an example we compute the spectrum of chiral primary states in symmetric $\operatorname{Spin}(5)_{R}$ representations. To facilitate the analysis we introduce and briefly discuss a new class of Lorentz non-invariant theories, which flow in the IR to the $(2,0)$ superconformal field theories but differ from them in the UV.
\end{abstract}

\section{Introduction}

Last year, a proposal for a light-cone quantization (or DLCQ) of M theory in terms of a quantum-mechanical system, called Matrix theory, appeared

\footnotetext{
${ }^{1}$ oferah@physics.rutgers.edu

${ }^{2}$ berkooz@ias.edu

${ }^{3}$ seiberg@ias.edu
} 
in [1]. The DLCQ (Discrete Light-Cone Quantization [2-5]) interpretation of this proposal appeared in [6], and a derivation of the conjecture was given in [7]. The idea that the large $N$ Matrix theory provides a light-cone Hamiltonian for $\mathrm{M}$ theory has passed many tests. However, the status of the conjecture that the finite $N$ theory provides a DLCQ of M theory is less clear, since direct comparisons of the low-energy limit of the finite $N$ theory with supergravity do not always work [8]. There is no compelling argument why a direct comparison with supergravity should work for finite $N$, since the low-energy limit of a DLCQ of M theory does not have to be equivalent to a DLCQ of supergravity $[9,10]$.

A simpler arena to analyze this question may be in DLCQ descriptions of field theories (see [11] for a review of works on this subject, and $[9,12]$ for recent works motivated by Matrix theory). The simplest case of such a description is the DLCQ proposed for $(2,0)$ superconformal theories in six dimensions (which were discovered in $[13,14]$ ). The DLCQ description involves a quantum-mechanical sigma model, and it was derived from Matrix theory in $[15,16]$. A generalization of this description to $(1,0)$ theories in six dimensions appeared in $[17,18]$, but we will not discuss it here.

In this paper we begin a detailed exploration of the DLCQ description of the $A_{k-1}(2,0)$ superconformal theories in six dimensions, which arise from the low-energy limit of $k$ coincident 5 -branes. One motivation for this study is the need to better understand the properties of Matrix-like DLCQ descriptions. The other motivation is the study of the $(2,0)$ superconformal theories in six dimensions, which are the simplest examples of non-trivial field theories above four dimensions. They are also interesting for applications to Matrix theory (see, e.g., [19]) and to field theory (it was conjectured in [20] that these theories may be related to the large $N$ limit of QCD). According to a conjecture of [21], they may also be related to M theory on $A d S_{7} \times S^{4}$.

In section 2 we describe the light-cone (and DLCQ) construction of $(2,0)$ field theories, and give a new derivation of it along the lines of [7]. In section 3 we analyze how the superconformal algebra looks in the light-cone frame, and see how it is realized in the quantum-mechanical description. The quantum mechanical description involves a sigma model on a singular space, and it seems that we need to resolve the singularities in order to be able to make sense of the model. In section 4 we describe such a resolution and its interpretation in terms of the space-time theory. In section 5 we analyze general properties of superconformal theories in DLCQ. In section 6 we compute the spectrum of chiral primary operators which are in symmetric representations of the Spin(5) R-symmetry, and interpret the chiral primary fields in terms of the natural coordinates parametrizing the moduli space of these theories. Section 7 includes a detailed analysis of the behavior of such chiral primary operators in some simple examples. We briefly outline 
a procedure to calculate $n$-point functions. The analysis of other primary operators, more complicated OPEs and explicit computations of higher $n$ point functions is left to future work [22].

As this paper was being completed, some overlapping results pertaining to the (free) theory of a single 5-brane appeared in [23], and a discussion of the superalgebra and of the single 5-brane theory appeared also in [24]. For a related discussion of the $(2,0)$ theories compactified on tori, see [25].

\section{The Quantum-Mechanical Light-Cone Descrip- tion}

In [15], a quantum-mechanical model was conjectured to give a light-cone (or DLCQ) description of the six dimensional field theory corresponding to the low-energy theory of $k \mathrm{M}$ theory 5 -branes. This includes the $A_{k-1}(2,0)$ superconformal theory as well as a decoupled free tensor multiplet. In this section we will review this model and its derivations, before going on to using it as a description of the spacetime theory in the rest of the paper.

\subsection{A Direct Derivation of the DLCQ Description}

In DLCQ, a light-like coordinate (which we will choose to be $x^{-}=\frac{1}{2}\left(x^{0}-x^{1}\right)$ ) is compactified on a circle of radius $R$, and the time coordinate is taken to be $x^{+}=\frac{1}{2}\left(x^{0}+x^{1}\right)$. The compactification of a light-like circle may be viewed as a limit of compactifications of near light-like circles, which in turn are equivalent (by a Lorentz boost) to compactifications of standard space coordinates. The light-like limit is obtained by taking the space-like circle to be very small, and looking at the theory of the modes which carry momentum around this circle. In general, one must also be careful to keep modes which correspond to finite energies in the original theory [7]. In our case this is relatively simple since the original theory we are starting with is conformal.

Thus, in order to obtain a DLCQ of the $(2,0)$ SCFTs we should look at their compactification on a circle of radius $R_{s}$ and take the limit $R_{s} \rightarrow 0$. At energies below the scale $1 / R_{s}$, the compactified theory is a $U(k)$ five dimensional $\mathcal{N}=2$ Super Yang-Mills theory, with a coupling constant $g_{5}^{2}=$ $R_{s}$, which goes to zero in the limit we are interested in. Note that this is very different from the behavior of standard field theories, which become strongly coupled when compactified on small circles, causing problems in their direct analysis in this way [9].

After the compactification, the momentum modes around the circle become instanton-like particles of the SYM theory [26] (namely, particles which are charged under the global current $J=*(F \wedge F)$ ). Finite energies in the original theory translate into very small velocities for these particles. Thus, 
in the limit $R_{s} \rightarrow 0$, the theory of $N$ of these modes, corresponding to a DLCQ with momentum $P_{-}=N / R$, reduces to a quantum mechanics on the moduli space of $N U(k)$ instantons embedded in $\mathbb{R}^{4}$, as in [15]. We will denote this moduli space by $\mathcal{M}_{N, k}$. For large $N$, this gives a light-cone description of the uncompactified $(2,0)$ theory.

This provides an alternative derivation of the light-cone (or DLCQ) description of [15]. However, the resulting theory we find here is not obviously well-defined, since the moduli space of instantons has singularities corresponding to small instantons. These singularities are not described just by the five dimensional $\mathcal{N}=2 \mathrm{SYM}$ theory, since this description breaks down at short distances (the theory is non-renormalizable). Thus, we need to add some information on how to regularize the singularities in the instanton moduli space. Another apparent problem is that the moduli space we find is non compact (in directions that do not correspond to space-time, in addition to the obvious non-compactness of the directions which are identified with space-time). This was interpreted in [15] as related to the IR behavior of the conformal theory, and this will be exemplified in detail in the following.

\subsection{A Derivation from M Theory with 5-branes}

One possible regularization is provided by the construction of the quantummechanical model as a limit of a DLCQ description of $M$ theory $[1,6]$ with 5 -branes, as described in [15]. One starts with a complete description of $\mathrm{M}$ theory in the presence of $k$ 5-branes [27], which is given by a quantum mechanical $U(N)$ SQCD theory with 8 (real) supercharges, with one adjoint hypermultiplet and $k$ hypermultiplets in the fundamental representation [28]. For finite gauge coupling $g_{Q M}$, this is a non-singular theory which is well-defined. The limit in which gravity decouples from the 5-brane theory $\left(M_{p} \rightarrow \infty\right)$ corresponds to the $g_{Q M} \rightarrow \infty$ limit of the quantum mechanical gauge theory, in which the Coulomb branch decouples from the Higgs branch. All the massive modes decouple in this limit, and the theory of the 5-branes is described by a supersymmetric sigma model on the Higgs branch of this theory ${ }^{4}$. It is a well-known fact [29] that this Higgs branch is exactly the moduli space of instantons $\mathcal{M}_{N, k}$ described above. In fact, the gauge theory gives the simplest construction of this moduli space, called the ADHM construction. It also provides for us a regularization, in the sense that we can, in principle, compute any space-time correlation function in the quantum mechanics for finite $g_{Q M}$, and then take the limit $g_{Q M} \rightarrow \infty$ and get the correlation functions of the superconformal theory. However, this regularization adds many more degrees of freedom than we actually need (in

\footnotetext{
${ }^{4}$ Note that since this is a conformal theory, we do not need to assume that the lowenergy limit of the DLCQ of M theory is the same as the DLCQ of supergravity.
} 
particular, it adds all of eleven dimensional supergravity). We will describe in section 4 a different regularization which is more useful for our purposes.

\subsection{A Description of the Model}

Let us now give a more concrete description of our theory. As we mentioned, the simplest description of the moduli space $\mathcal{M}_{N, k}$ is as the Higgs branch of a $U(N)$ gauge theory with 8 supercharges, an adjoint hypermultiplet and $k$ fundamental hypermultiplets. Let us denote the scalar components of these hypermultiplets by $X, \tilde{X}$ (two complex scalars in the adjoint representation of $U(N))$ and by $q_{i}, \tilde{q}^{i}$ in the $\mathbf{N}$ and $\overline{\mathbf{N}}$ representations of $U(N)(i=1, \cdots, k)$. The Higgs branch is parametrized by the values of these fields, subject to the constraints enforcing the vanishing of the scalar potential :

$$
\left[X, X^{\dagger}\right]-\left[\tilde{X}, \tilde{X}^{\dagger}\right]+q_{i} q_{i}^{\dagger}-\left(\tilde{q}^{i}\right)^{\dagger}\left(\tilde{q}^{i}\right)=0
$$

and

$$
[X, \tilde{X}]+q_{i} \tilde{q}^{i}=0,
$$

and modded out by the $U(N)$ gauge symmetry. The total (real) dimension of this space is $4 N k+4 N^{2}-3 N^{2}-N^{2}=4 N k$, and it is a hyperKähler manifold.

The equations (and gauge symmetry) do not act on 4 decoupled coordinates which are $\operatorname{tr}(X), \operatorname{tr}(\tilde{X})$. Thus, the moduli space decomposes as $\mathcal{M}_{N, k}=\mathbb{R}^{4} \times \mathcal{M}_{N, k}^{0}$. The decoupled $\mathbb{R}^{4}$ part will give rise to eight nonlinearly realized supersymmetries, which act by shifting the corresponding fermions. It will sometimes be convenient to denote the coordinates of the $\mathbb{R}^{4}$ component by $\tilde{x}$, and those of $\mathcal{M}_{N, k}^{0}$ by $v$.

There is a natural metric on the moduli space, which is the hyperKähler metric. From the gauge theory point of view this is the classical metric on the Higgs branch, which is not renormalized. On the total space of fields, we can define a scalar function

$$
K=\left|q_{i}\right|^{2}+\left|\tilde{q}^{i}\right|^{2}+|X|^{2}+|\tilde{X}|^{2}
$$

given by the sum of the absolute value squared of all scalar fields. The restriction of this function to the moduli space defines a scalar function on the moduli space, which we will also denote by $K$. Then, the metric on the moduli space is $g_{i j}=\frac{1}{2} \partial_{i} \partial_{j} K$ where $i, j$ label the coordinates of the moduli space. Note that the space is scale-invariant.

The global symmetries of the theory are $S U(2)_{R} \times S U(2)_{L} \times \operatorname{Spin}(5) \times$ $U(k)$. The first two factors correspond to the rotation symmetries inside the 5-brane transverse to the light-cone coordinate, the third factor is the 
rotations transverse to the 5-brane (or, equivalently, the R-symmetry of the $\mathcal{N}=(2,0)$ supersymmetry in spacetime), and the last factor corresponds to the gauge symmetry of the $(2,0)$ theory after it is compactified on a circle. The supercharges in the quantum mechanics are in the $(\mathbf{2}, \mathbf{1}, \mathbf{4}, \mathbf{1})$ representation of this group, so that $S U(2)_{R} \times \operatorname{Spin}(5)$ is the R-symmetry of the quantum mechanics. The fields described above are in the following representations :

$\begin{array}{cccccc} & U(N) & S U(2)_{R} & S U(2)_{L} & S p i n(5) & U(k) \\ X_{H} & \mathbf{N}^{2} & \mathbf{2} & \mathbf{2} & \mathbf{1} & \mathbf{1} \\ \Theta_{X} & \mathbf{N}^{2} & \mathbf{1} & \mathbf{2} & \mathbf{4} & \mathbf{1} \\ q_{H} & \mathbf{N} & \mathbf{2} & \mathbf{1} & \mathbf{1} & \mathbf{k} \\ \psi_{q} & \mathbf{N} & \mathbf{1} & \mathbf{1} & \mathbf{4} & \mathbf{k}\end{array}$

where $X_{H}$ denotes the scalars in the adjoint hypermultiplets $(X$ and $\tilde{X}), q_{H}$ denote the scalars in the fundamental hypermultiplets $(q$ and $\tilde{q}), \Theta_{X}$ are the fermionic partners of $X, \tilde{X}, \psi_{q}$ are the fermionic partners of $q, \tilde{q}$, and all the fields obey appropriate reality conditions. The bosonic coordinates are all neutral under the $\operatorname{Spin}(5)$, while the fermionic coordinates are all in the $\mathbf{4}$ representation. The other global symmetries act on the moduli space in a non-trivial way (except for $S U(2)_{R}$ they commute with supersymmetry).

\section{The Superconformal Algebra in Spacetime and in the Quantum Mechanics}

\subsection{Bosonic Components in the Spacetime Algebra}

The bosonic part of the superconformal algebra in six dimensions includes the $S O(6,2)$ conformal algebra

$$
\left[M_{\alpha \beta}, M_{\gamma \delta}\right]=-i\left(\eta_{\alpha \gamma} M_{\beta \delta}+\eta_{\beta \delta} M_{\alpha \gamma}-\eta_{\alpha \delta} M_{\beta \gamma}-\eta_{\beta \gamma} M_{\alpha \delta}\right),
$$

where $\alpha, \beta=0, \cdots, 7, \eta_{\alpha \beta}=\operatorname{diag}\left(-1,1^{6},-1\right)$.

The components $M_{i j}$ of this algebra $(i, j=0, \cdots, 5)$ are identified with the usual Lorentz generators, while the other components are related to the standard conformal generators by

$$
P_{i}=M_{6 i}+M_{7 i} ; \quad K_{i}=M_{6 i}-M_{7 i} ; \quad D=M_{67} .
$$

Here $P_{i}$ is the translation generator, $K_{i}$ generates special conformal transformations and $D$ generates dilatations. With these identifications, (5) leads 
to the usual conformal algebra

$$
\begin{array}{r}
{\left[M_{i j}, P_{k}\right]=-i\left(\eta_{i k} P_{j}-\eta_{j k} P_{i}\right) ;\left[M_{i j}, K_{k}\right]=-i\left(\eta_{i k} K_{j}-\eta_{j k} K_{i}\right)} \\
{\left[M_{i j}, M_{k l}\right]=-i \eta_{i k} M_{j l} \pm \text { permutations } ;\left[M_{i j}, D\right]=0 ; \quad\left[D, K_{i}\right]=i K_{i}} \\
{\left[D, P_{i}\right]=-i P_{i} ;\left[P_{i}, K_{j}\right]=-2 i M_{i j}+2 i \eta_{i j} D}
\end{array}
$$

Our description of this theory in discrete light cone quantization sees only a sector of the theory with fixed $P_{-}=P_{0}-P_{1}=N / R$. Thus, in the quantum mechanical description we should only be able to see those elements of the superconformal algebra that commute with $P_{-}$. These include the Galilean generators $M_{i j}, P_{i}, H=P_{+}=P_{0}+P_{1}$ and $V_{i}=M_{0 i}-M_{1 i}$ (where now $i, j=2,3,4,5)$. Two other elements of the conformal algebra also commute with $P_{-}$: they are $K_{-}=K_{0}-K_{1}$ and $T=D-M_{01}$. $T$ has a natural interpretation as a dilatation followed by a boost in the $x^{1}$ direction, which is needed to cancel the effect of the dilatation on $P_{-}$.

The non-zero commutation relations of these elements are :

$$
\begin{array}{r}
{\left[M_{i j}, P_{k}\right]=-i\left(\eta_{i k} P_{j}-\eta_{j k} P_{i}\right) ; \quad\left[M_{i j}, V_{k}\right]=-i\left(\eta_{i k} V_{j}-\eta_{j k} V_{i}\right) ;} \\
{\left[P_{i}, V_{j}\right]=-i \eta_{i j} P_{-} ; \quad\left[T, P_{i}\right]=-i P_{i} ; \quad\left[T, V_{i}\right]=i V_{i} ; \quad\left[P_{i}, K_{-}\right]=2 i V_{i} ;} \\
{\left[H, V_{i}\right]=-2 i P_{i} ; \quad\left[H, K_{-}\right]=-4 i T ; \quad[T, H]=-2 i H ; \quad\left[T, K_{-}\right]=2 i K_{-} .}
\end{array}
$$

Note the explicit appearance of $P_{-}$in the commutation relations as a central term. The analysis of the bosonic part of the superconformal algebra does not depend on the dimension of space-time, and we expect to find the same algebra (10) in a DLCQ description of any conformal theory. Of course, the fermionic part described below will depend strongly on the fact that we are in $5+1$ dimensions.

\subsection{Fermionic Components of the Superconformal Algebra}

The $(2,0)$ superconformal algebra in six dimensions includes 32 fermionic generators, which are all $S O(6,2)$ spinors of the same chirality (say, $\mathbf{8}_{\mathbf{s}}$ ) and are a fundamental 4 of $S p(2) \simeq \operatorname{Spin}(5)$. Their commutation relation is of the form

$$
\left\{Q_{\alpha}^{i}, Q_{\beta}^{j}\right\}=J^{i j}\left(\Gamma^{\mu \rho}\right)_{\alpha \beta} M_{\mu \rho}+\delta_{\alpha \beta} R^{i j},
$$

where $J$ is the anti-symmetric form of $S p(2), R$ is the charge of the $S p(2)$ current, and $\Gamma^{\mu \rho}=\frac{1}{2}\left[\Gamma^{\mu}, \Gamma^{\rho}\right]$. 
It will be convenient to decompose the $Q$ operators according to their charges under the $S O(1,1)$ symmetries rotating $x^{0}, x^{1}$ and $x^{6}, x^{7}$, or, equivalently, according to the eigenvalues of $\Gamma^{0} \Gamma^{1}$ and $\Gamma^{6} \Gamma^{7}$ in the spinor representation of $S O(6,2)$. Defining

$$
\Gamma^{ \pm}=\frac{1}{2}\left(\Gamma^{0} \pm \Gamma^{1}\right) ; \quad \hat{\Gamma}^{ \pm}=\frac{1}{2}\left(\Gamma^{6} \pm \Gamma^{7}\right)
$$

the decomposition into eigenstates $Q, \tilde{Q}, S$ and $\tilde{S}$ satisfies

$$
\begin{array}{cccc} 
& \Gamma^{0} \Gamma^{1} & \Gamma^{6} \Gamma^{7} & \\
S & 1 & 1 & \Gamma^{+} S=\hat{\Gamma}^{+} S=0 \\
\tilde{S} & -1 & 1 & \Gamma^{-} \tilde{S}=\hat{\Gamma}^{+} \tilde{S}=0 \\
Q & -1 & -1 & \Gamma^{-} Q=\hat{\Gamma}^{-} Q=0 \\
\tilde{Q} & 1 & -1 & \Gamma^{+} \tilde{Q}=\hat{\Gamma}^{-} \tilde{Q}=0
\end{array}
$$

Then, the commutation relations (13) are schematically of the form (neglecting the $\mathrm{R}$ charge contributions, and without writing down the indices, which follow from the symmetries):

$$
\begin{aligned}
& \{Q, Q\} \sim H ; \quad\{Q, \tilde{Q}\} \sim P_{i} ; \quad\{\tilde{Q}, \tilde{Q}\} \sim P_{-} ; \\
& \{Q, \tilde{S}\} \sim M_{0 i}+M_{1 i} ; \quad\{\tilde{Q}, S\} \sim V_{i} ; \\
& \{Q, S\} \sim M_{i j}^{R}-T ; \quad\{\tilde{Q}, \tilde{S}\} \sim M_{i j}^{L}-\left(M_{01}+D\right) \\
& \{S, S\} \sim K_{-} ; \quad\{S, \tilde{S}\} \sim K_{i} ; \quad\{\tilde{S}, \tilde{S}\} \sim K_{+}
\end{aligned}
$$

where we have decomposed the $S O(4)$ generators $M_{i j}$ into $S U(2)_{R}$ generators $M_{i j}^{R}$ and $S U(2)_{L}$ generators $M_{i j}^{L}$.

\subsection{Identification of the Bosonic Elements in the Quantum Mechanics}

Let us now try to identify the algebra (10) in our quantum mechanical description. This description includes four variables $\tilde{x}^{i}$ which are free and decoupled, corresponding to the center of mass position. The other $(4 N k-4)$ variables $v^{k}$ are coordinates on a non-trivial hyperKähler manifold. The metric on the target space of the quantum mechanical sigma model is of the form $g_{i j}=\frac{1}{2} \partial_{i} \partial_{j} K$ where the function $K$ was described in the previous section (the inverse metric will be denoted by $g^{k l}, g^{k l} g_{l j}=\delta_{j}^{k}$ ). We will denote all $4 N k$ coordinates by the collective name $x^{i}=\left\{\tilde{x}^{j}, v^{k}\right\}$. The conjugate momenta to these variables will be denoted by $\Pi_{x^{i}}\left(\left[\Pi_{x^{i}}, x^{j}\right]=-i \delta_{i}^{j}\right)$. Their action on wave functions is the same as a derivative, $\Pi_{x} \sim-i \partial_{x}$. 
The identification of the Galilean subalgebra of the superconformal algebra is mostly straightforward. The operator $H$ is just the Hamiltonian of the quantum mechanical sigma model

$$
H=\frac{R}{N}\left(\delta_{i j} \Pi_{\tilde{x}^{i}} \Pi_{\tilde{x}^{j}}+g^{k l} \Pi_{v^{k}} \Pi_{v^{l}}\right)+\text { fermions. }
$$

Note that we chose the Hamiltonian to be explicitly proportional to $R / N$. This is the natural scaling for the interpretation of this Hamiltonian as the DLCQ of a space-time theory, since then $H=P_{+}=\left(P_{i}^{2}+M^{2}\right) / P_{-}=$ $R\left(P_{i}^{2}+M^{2}\right) / N$. The natural scaling for the quantum-mechanical sigmamodel Hamiltonian does not include this factor of $R / N$. However, since the sigma model is scale invariant, the two conventions differ only by rescaling all coordinates and momenta by $\sqrt{R / N}$. In particular, correlation functions in the sigma model will be functions of $\sqrt{\frac{N}{R}} \cdot x$, and not of $x$ and $R / N$ separately.

The momenta $P_{i}$ and boosts $V_{i}$ act only on the center of mass coordinates, so we can identify them with

$$
P_{i}=\Pi_{\tilde{x}^{i}} ; \quad V_{i}=P_{-} \tilde{x}^{i} .
$$

The rotations $M_{i j}$ generate an $S O(4) \simeq S U(2)_{R} \times S U(2)_{L}$ global symmetry in the quantum mechanics, which acts in a rather complicated way on the sigma model coordinates. These operators satisfy the commutation relations (10).

Next, we should identify the operators $T$ and $K_{-}$. For this we note that (using (10)) we may identify $H, \frac{1}{2} T$ and $\frac{1}{4} K_{-}$with generators of an $S O(1,2)$ algebra. We can identify this algebra with the conformal algebra of the quantum mechanical theory ${ }^{5}$, generated by $\delta t=\epsilon_{1}+\epsilon_{2} t+\epsilon_{3} t^{2}$. In the case at hand, we may explicitly write these as

$$
\begin{aligned}
T & =-\left(\frac{1}{2} g^{i j} \frac{\partial K}{\partial x^{i}} \Pi_{x^{j}}-2 i N k\right)=-\frac{1}{4} g^{i j}\left(\frac{\partial K}{\partial x^{i}} \Pi_{x^{j}}+\Pi_{x^{i}} \frac{\partial K}{\partial x^{j}}\right) \\
K_{-} & =-\frac{1}{4} P_{-} g^{i j} \frac{\partial K}{\partial x^{i}} \frac{\partial K}{\partial x^{j}}
\end{aligned}
$$

up to additional fermionic terms. The expressions above are meaningful only away from the singularities of the manifold, where additional contributions may be localized. In the conformal quantum mechanics, these operators obey the commutation relations (10). Note that $K_{-}$vanishes only at the origin of the moduli space $(x=0)$.

\footnotetext{
${ }^{5}$ Using the same conventions as (5) and (6) for the conformal algebra of the quantum mechanics, $H \sim\left(P_{0}\right)_{Q M}, T \sim 2 D_{Q M}$ and $K_{-} \sim 4\left(K_{0}\right)_{Q M}$.
} 
Obviously, these identifications are meant to be relevant only at some specific time, say $t=0$. The operators may then be evolved in time in the usual way, by the equation $\mathcal{O}(t)=e^{i t H} \mathcal{O}(0) e^{-i t H}$. For instance, $V_{i}(t) \simeq$ $P_{-} \tilde{x}^{i}+2 t \Pi_{\tilde{x}^{i}}$. Note that we expect to find $H=P_{+}=\left(\left(P_{i}\right)^{2}+M^{2}\right) / P_{-}$, so we may identify the second ( $v$-dependent) part of $H$ with $M^{2}$ in spacetime (which is a Casimir operator of the Lorentz algebra but not of the conformal algebra).

\subsection{Identification of the Fermionic Operators in the Quan- tum Mechanics}

It is easy to identify also most of the operators described in $\S 3.2$ in the quantum mechanical description. The charges of the $S p(2)$ R-currents in spacetime may be identified with the $S p(2)$ R-charges of the quantum mechanics, which involve only the fermions. Using the commutation relations (16) it is easy to check that $Q, \tilde{Q}$ and $S$ commute with $P_{-}$, while $\tilde{S}$ does not, so we do not expect to identify $\tilde{S}$ in the quantum mechanical description.

The operators $Q$ and $\tilde{Q}$ are simply the spacetime supersymmetry generators. The $Q$ 's are the generators which are linearly realized in the quantum mechanics as the 8 supercharges of the quantum mechanics. Schematically they are of the form $Q_{a}^{\alpha} \sim \sqrt{R / N} \Theta_{a}^{i} \Pi_{x_{\alpha}^{i}}$, where we divided the target space coordinates into $S U(2)_{R}$ doublets. $\tilde{Q}$ is non-linearly realized in the quantum mechanics, and schematically it is given by $\tilde{Q}_{a}^{\dot{\alpha}} \sim 2 \sqrt{P_{-}} \Theta_{a}^{\dot{\alpha}}$, where $\Theta_{a}^{\dot{\alpha}}$ is the superpartner of $\tilde{x}^{i}$ (denoted by $\operatorname{tr}\left(\Theta_{X}\right)$ above). $\tilde{Q}$ is in the $(\mathbf{1}, \mathbf{2}, \mathbf{4}, \mathbf{1})$ representation of the global symmetry, while $Q$ and $S$ are in the $(\mathbf{2}, \mathbf{1}, \mathbf{4}, \mathbf{1})$ representation.

The superconformal generator $S$ may be identified in the quantum mechanics (up to a constant) with the other fermionic generator of the quantum mechanical superconformal algebra. Schematically, it is given by $S_{a}^{\alpha} \sim$ $\sqrt{P_{-}} \Theta_{a}^{i} \frac{\partial K}{\partial x_{\alpha}^{i}}$. It is easy to check that with these identifications, the commutation relations (16) are obeyed. The $Q$ 's and $S$ 's form doublets of the $S O(1,2)$ algebra mentioned above $\left([T, Q] \sim-i Q,[T, S] \sim i S,\left[K_{-}, Q\right] \sim-i S,[H, S] \sim\right.$ $\left.-i Q,\left[K_{-}, S\right]=[H, Q]=0\right)$.

To summarize, we have found that our quantum mechanical sigma model is actually a superconformal sigma model. This is consistent with the fact that the R-symmetry group is $S U(2)_{R} \times \operatorname{Spin}(5)$, which is one of the possible $\mathrm{R}$-symmetries for superconformal quantum mechanics [30]. The superconformal algebra of the quantum mechanical theory includes the generators $H=P_{+}, T, K_{-}, Q$ and $S$ of the space-time superconformal algebra, all of which commute with $P_{-}$. The other generators which commute with $P_{-}$ 
are realized either as charges of the $S U(2)_{R} \times S U(2)_{L} \times S O(5)$ global symmetries, or (in the case of $P_{i}, V_{i}$ and $\tilde{Q}$ ) as simple operators acting on the decoupled free fields corresponding to the center of mass position.

\section{Resolution of the singularities}

\subsection{The need for the resolution}

It is natural to organize the states of the space-time superconformal theory into representations of the superconformal algebra. The representations which appear in physical theories include primary states, which are annihilated by all the special conformal generators $K_{\mu}$ and $S$ (we will elaborate more on this in section 5 ). Since we identified $K_{-}$with the special conformal generator of the quantum mechanical theory, such states will correspond to primary states in the quantum mechanics ${ }^{6}$, which are annihilated by $K_{-}$.

From the form of $K_{-}$given above, it is clear that such a state must be concentrated completely at the origin of moduli space. Other local operators, obtained by acting with $P$ and $Q$ on the primary operator, will also be concentrated there. The fact that local operators in space-time correspond to states in the quantum mechanics which are localized at the origin of the moduli space had been anticipated by L. Susskind [31] based on the following intuitive picture. We are interested in local operators in spacetime, which are a disturbance only in a very small region of space-time. Thus, their wave function should certainly be localized in the quantum mechanical variables which correspond to space-time positions. These arise from the $X$ fields in the gauge theory description. The other variables in the quantum mechanics, arising from the $q$ fields, are associated with the instanton size. Large instantons are not expected to correspond to local operators, so we expect states corresponding to local operators to be localized at $q=\tilde{q}=0$.

Using the algebra, one can arrive at the same conclusion in another way. The way to obtain a local operator in space-time is to create a disturbance in a compact region of space-time and then shrink it using the dilatation operator. The corresponding action in the quantum mechanics will be to shrink all the support of any wave function to the origin.

There is one obvious wave function of this type, which is just $\prod_{i} \delta\left(x^{i}\right)$, but since the origin is very singular it is hard to say if there are not more states that are "hidden inside the singularities." Thus, we would like to be able to resolve the singularities and deal with a smooth manifold. Luckily, there exists a simple resolution of the space which makes it completely smooth.

\footnotetext{
${ }^{6}$ States that are not primary in space-time may also appear as primary in the quantum mechanics, since the $0+1$ dimensional superconformal algebra is smaller.
} 
In this section we will describe this resolution and its physical interpretation in space-time.

As described in section 2, the space $\mathcal{M}_{N, k}$ is the Higgs branch of a gauge theory with 8 supercharges, which is the space of $X$ 's and $q$ 's subject to (1) and (2) and modded out by the gauge group. This space is singular whenever the gauge group is not completely broken, since then there are additional massless fields in the gauge theory. From the point of view of the instanton moduli space these singularities correspond to small instantons. By adding a Fayet-Iliopoulos term to the gauge theory, we can force the gauge group to be completely broken at all points on the Higgs branch, and then the Higgs branch is no longer singular. The Fayet-Iliopoulos parameter consists of 3 real scalars, which are a triplet of $S U(2)_{R}$. They appear on the right side of the equations (1) and (2). By an $S U(2)_{R}$ transformation we can always choose just one of these scalars to be non-zero. The Higgs branch of the theory, which is the deformed moduli space $\tilde{\mathcal{M}}_{N, k}$, is then given by the space of $X$ 's and $q$ 's subject to the equations

$$
\left[X, X^{\dagger}\right]-\left[\tilde{X}, \tilde{X}^{\dagger}\right]+q_{i} q_{i}^{\dagger}-\left(\tilde{q}^{i}\right)^{\dagger}\left(\tilde{q}^{i}\right)=\zeta I_{N}
$$

and

$$
[X, \tilde{X}]+q_{i} \tilde{q}^{i}=0,
$$

modded out by the gauge group, where $\zeta$ is some non-zero real number and $I_{N}$ is the $N \times N$ unit matrix.

From the mathematical viewpoint turning on $\zeta$ is a compactification of the small-instanton region of the moduli space. This compactification was used in [32] to compute partition functions of $\mathcal{N}=44 \mathrm{D}$ gauge theories, and in [33] to compute the cohomology of $\mathcal{M}_{N, k}$. It has no known interpretation in terms of instantons, but it is natural in the context of their generalization to stable sheaves in algebraic geometry.

\subsection{Spacetime Interpretation of the Resolution}

What is the physical interpretation of the parameter $\zeta$ in the space-time theory? We can discuss this question within the theory of the 5-branes alone, or we can do so within the $U(N)$ quantum mechanics corresponding to the full $\mathrm{M}$ theory with the 5 -branes, which flows in the IR (on one of its branches) to the 5-brane theory. We will use the latter, more general, description. Apriori, not every deformation of the quantum mechanics corresponds to a change in the theory in space-time, as the space-time interpretation may collapse. We do not expect this, however, to happen in our case.

Strictly speaking, the deformation by $\zeta$ lifts the Coulomb branch, which corresponds to 0 -branes moving away from the longitudinal 5 -brane. It does 
so, however, in a very limited way. Unlike its pronounced effect on the origin of the Higgs branch and of the Coulomb branch, its effect far out along the Coulomb branch is merely an addition of a constant to the DLCQ Hamiltonian. As such, it changes the dynamics there only by an insignificant phase. We therefore still expect to have, for example, bound states corresponding to gravitons [34]. We have not lost the space-time interpretation, and the deformation by $\zeta$ should have such an interpretation.

We will argue that the space-time interpretation of the parameter $\zeta$ is that of turning on a constant 3 -form field $C$ in $\mathrm{M}$ theory. This field is not gauge invariant, and can be gauged away in the absence of 5-branes. However, in the presence of a 5-brane, gauge transformations of the $C$ field in the bulk act also on the self-dual 3-form field strength $H$ living on the 5 -brane, and the gauge invariant (self-dual) field on the 5-brane is actually $(H-C)$ [35]. Thus, by a gauge transformation we can turn the 3 -form field $C$ in the bulk into a constant (self-dual) 3-form field strength $H$ on the 5-brane. The component of the $H$ field that we turn on is $H_{+i j}$, where the $i, j$ indices are in the non-compact directions of the 5-brane and the + index is the DLCQ time direction. In most of the analysis below we will use this gauge freedom and discuss the space-time theory with an $H$-field turned on (we can only do this explicitly for the case of a single 5-brane, but this should not change our results).

To get the precise coupling we can follow the procedure of [7]. We will do so with some detail. At the first step we are interested in going from $\mathrm{M}$ theory on an almost light-like circle, which we will denote by $M$, to $\mathrm{M}$ theory on a space-like circle with radius $R_{s}$, a configuration which we will denote by $M_{s}$ (we will label quantities in $M_{s}$ by a subscript $s$ ). We will then go to the $\widetilde{M}$ system by an appropriate rescaling.

In the original $M$ system, on a light-like circle, we wish to set $H_{+i j}$ to some non-zero value. The DLCQ Hamiltonian is of the general form

$$
P_{+}=R M_{p}^{2} F\left(\frac{P_{i}}{M_{p}}, \frac{H_{+i j}}{R M_{p}^{4}}\right),
$$

for some function $F$. This form is determined on dimensional grounds, and by the requirement that the DLCQ Hamiltonian be covariant under longitudinal boosts. Under a longitudinal boost the dynamical quantities change according to their $M_{01}$ quantum numbers, but we also change the value of $R$. The quantity $H_{+i j} / R$ remains invariant under such a boost, and, therefore, it is the correct one to enter the function $F$. The factors of $M_{p}$ are then fixed by dimensionality. Our final goal is to go over to the $\widetilde{M}$ system in a way that preserves the physics, as encoded by $F$.

Next, as in [7], we make the circle slightly space-like and then boost the system to the configuration $M_{s}$, in which $\mathrm{M}$ theory is compactified on a 
spatial circle with radius $R_{s}$. When we deform the circle to be slightly spacelike we do not change $H$ and preserve the fact that $H_{-i j}=0$. The reason we must do this is that the self-duality condition on the 3-form field $H$ implies that the physical components of $H_{+i j}$ transform as a triplet of $S U(2)_{R}$ (and a singlet of $\left.S U(2)_{L}\right)$, while those of $H_{-i j}$ transform as a triplet under $S U(2)_{L}$ (and a singlet of $\left.S U(2)_{R}\right)^{7}$. This is true irrespectively of details of the circle on which we compactify. As our deformation has (by construction) only $S U(2)_{R}$ quantum numbers, we need to maintain that $H_{-i j}=0$.

Now, when we go to the $M_{s}$ system after the boost, we find that

$$
H_{s,+i j}=\frac{R_{s}}{R} H_{+i j}, H_{s,-i j}=0,
$$

and that the Hamiltonian is now of the form

$$
\left(P_{0}\right)_{s}=R_{s} M_{p}^{2} F\left(\frac{P_{i}}{M_{p}}, \frac{H_{s,+i j}}{R_{s} M_{p}^{4}}\right) .
$$

In order to go to $\widetilde{M}$, we now want to rescale time and distances such that the energy of the physical processes we are interested in remains fixed, and the relevant physics remains the same. This criterion determines that, in going to $\widetilde{M}$, we need to keep the arguments of the function $F$ fixed. In [7] this determined the scaling $\frac{P_{i}}{M_{p}}=\frac{\tilde{P}_{i}}{\tilde{M}_{p}}$. Here it also determines the relation

$$
\frac{H_{s,+i j}}{R_{s} M_{p}^{4}}=\frac{\tilde{H}_{+i j}}{R_{s} \tilde{M}_{p}^{4}} .
$$

Once we are in the $\widetilde{M}$ system, and have determined all the quantities in this system, we can go over to the IIA description (which is valid since the radius $R_{s}$ of the space-like circle in this system is small) and write down the Lagrangian for the 0 -branes. $\tilde{H}_{+i j}$ may now be interpreted as a non-zero field strength on the 4-brane, with a specific chirality ${ }^{8}$. Such a field enters the Lagrangian of the D0-branes as a FI term. This can be seen in several ways. For instance, a computation in the free conformal field theory of the 0-4 strings shows that a field strength shifts the mass of the $0-4$ strings in the same way that a FI term does.

A more intuitive way is to perform a gauge transformation on the bulk fields in the string theory, such that the constant field strength $F$ on the D4-brane becomes a constant NS B-field in the bulk (this is similar to the

\footnotetext{
${ }^{7}$ Recall that we decomposed the $S O(4)$ rotation symmetry of the 5-brane transverse to the light-cone direction into $S U(2)_{R} \times S U(2)_{L}$.

${ }^{8}$ When we wrap the 5 -brane on a circle of radius $R_{s}$ in the $x^{1}$ direction to get a D4brane [35], the $4+1$ dimensional field strength on the D4-brane is $F_{i j}=R_{s} H_{1 i j}$.
} 
inverse of the gauge transformation we used above to turn $C$ into $H$ ). The low-energy action of D-branes does not contain just the $\operatorname{tr} F^{2}$ term, but rather $\operatorname{tr}(F-B)^{2}$, where $B$ is the pullback of the space-time NS 2-form field to the D-brane worldvolume. If we start with the action for a wrapped 2-brane and T-dualize to go to the action of 0-branes, this becomes a term of the form $\operatorname{tr}\left(\left[X_{\mu}, X_{\nu}\right]-B_{\mu \nu}\right)^{2}$, and again we see that some of the components of the $B$ field appear as a FI term.

Going back to the quantum mechanical Lagrangian for the D0-branes in the $\widetilde{M}$ system, we find that it is of the schematic form (suppressing indices)

$$
\tilde{L}=\frac{1}{R_{s}} \dot{\tilde{X}}^{2}+R_{s} \tilde{M}_{p}^{6}\left([\tilde{X}, \tilde{X}]+\frac{\tilde{H}_{+}}{R_{s} \tilde{M}_{p}^{6}}\right)^{2} .
$$

Using the relation to the original M-theory variables [7]

$$
X=\left(\frac{R}{R_{s}}\right)^{\frac{1}{2}} \tilde{X}, M_{p}^{2} R=\tilde{M}_{p}^{2} R_{s}
$$

and

$$
\frac{\tilde{H}_{+}}{R_{s} \tilde{M}_{p}^{4}}=\frac{H_{s,+}}{R_{s} M_{p}^{4}}=\frac{H_{+}}{R M_{p}^{4}},
$$

one obtains

$$
L=\frac{1}{R} \dot{X}^{2}+R M_{p}^{6}\left(\left[X_{i}, X_{j}\right]+\frac{H_{+i j}}{R M_{p}^{6}}\right)^{2} .
$$

Thus, we identify the Fayet-Iliopoulos term $\zeta$ with $\frac{H_{+i j}}{R M_{p}^{6}}$, or more generally with $\frac{C_{+i j}}{R M_{p}^{6}}$, in the space-time $\mathrm{M}$ theory that we are describing ${ }^{9}$.

Note that our identification of the Fayet-Iliopoulos term with the $C_{+i j}$ field in space-time is completely general, and can be used even in the absence of any 5-branes. Of course, in such a case this field may locally be completely gauged away. This is consistent with the fact that in the absence of any charged matter ( $q$ 's), the Fayet-Iliopoulos term only contributes a constant to the Hamiltonian, which does not change the dynamics. In the presence of 5 -branes and the constant $C$-field, we find that particles with longitudinal momentum are attracted to the 5-branes, since the minimal energy away from the 5 -branes (where $q=0$ ) is $|\zeta|^{2}$, while inside the 5-branes it is zero (for configurations satisfying (20) and (21)).

\footnotetext{
${ }^{9}$ This was recently noted also in $[36-38]$.
} 


\subsection{New Lorentz Non-Invariant Theories}

In the previous subsection we described the role of the FI term in the full theory of $\mathrm{M}$ theory with $k 5$-branes, described by the $U(N)$ gauge quantum mechanics. However, in this paper we are actually interested in taking $M_{p} \rightarrow$ $\infty$, in order to decouple the $(2,0)$ field theory on the 5 -brane from the bulk. Since, as mentioned above and described in more detail below, we would like to use $\zeta$ as a UV cutoff for the quantum mechanical sigma model that we obtain, we would like to take this limit such that the FI term remains fixed. However, since we found above that $\zeta \simeq \frac{C_{+i j}}{R M_{p}^{6}}$, we need to take $C_{+i j} \propto$ $M_{p}^{6} \rightarrow \infty$ in this limit. Thus, the deformed quantum mechanical theory (the sigma model on the deformed moduli space $\tilde{\mathcal{M}}_{N, k}$ ) describes in spacetime the theory of $k$-branes as $M_{p} \rightarrow \infty$, with a $C$-field which also goes to infinity as $C \propto M_{p}^{6}$. Obviously, such a limit is not accessible from the low-energy supergravity theory (for a single 5-brane we can translate the $C$ field into an $H$ field whose energy density is very large in Planck units), but in principle it exists in M theory, and our DLCQ description provides a construction of the resulting theory.

The resulting theory is not equivalent to the $(2,0)$ field theory, as one clearly sees from the fact that its DLCQ description is quite different (obviously, it is not Lorentz invariant, since we explicitly broke the $S U(2)_{R}$ which was part of the space-time Lorentz group). Many of the UV properties of the resulting theory will be different from those of the $(2,0)$ theory (as one can compute explicitly using the quantum mechanics), but it will still flow to the $(2,0)$ theory in the IR. This may be deduced from the fact that the change induced by the FI term is particularly important near the origin of the Higgs branch, and is less important far along the flat directions (thus, the long distance behavior of the new theory is governed by the $(2,0)$ fixed point). This also shows that it is incorrect to think of this theory as a mere change of the vacuum of the $(2,0)$ field theory, as this would not modify the theory in the UV. Instead, one can think of this as a change in the short-distance (UV) properties of this theory which does not change its long-distance properties.

To summarize, our construction naturally implies the existence of a new class of Lorentz non-invariant theories, whose UV properties are very different from those of the standard field theories (the theory is not Lorentz invariant nor scale invariant in the UV $)^{10}$. Even though we have not encountered such theories before, and we do not seem to be able to access any of their properties through the low-energy supergravity theory ${ }^{11}$, the preceding

\footnotetext{
${ }^{10}$ Note that these theories are not Lorentz invariant but still have 16 supercharges. They are similar in this sense to the theories recently discussed in [36].

${ }^{11}$ For $k=1$ and for values of $C$ (or $H$ ) that are small in Planck units, it should be possible to explicitly compute the effect of turning on this field also directly in spacetime $[24,35,39]$. However, it is not known how to do this for $k>1$, and in any case we are
} 
construction is (up to possible subtleties of the large $N$ limit) a complete calculable definition of these theories.

\section{4 $\zeta$ as an Ultra-Violet Cutoff}

As an ultra-violet cutoff $\zeta$ appears in the theory in a familiar and straightforward way. $\zeta$ has negative mass dimension $(-2)$, so taking $\zeta$ to be small is equivalent to going to large time differences. When analyzing the theory with finite $\zeta$, we will usually be interested only in the long-time behavior of correlation functions. At long times the quantum mechanical theory will flow to a superconformal theory, which will contain the information about the superconformal theory in space-time.

The procedure of extracting $n$-point functions from the theory with the cut-off is standard. The key point is that we are interested in the behavior only as $t \rightarrow \infty$. By appropriate wave-function renormalization we will extract the quantities that remain finite as we take the UV cut-off to infinity or, equivalently, flow to the $\mathrm{IR}^{12}$. All other quantities, with a non-trivial dependence on the cut-off, will be interpreted as artifacts of the cut-off.

Let us briefly demonstrate this standard procedure for 2 and 3-point functions. The $x$ dependence of wave functions can always be chosen to be through the combination $x / \sqrt{\zeta}$. Then, the time dependence will always appear through the combination $\zeta / R t$. Given a wave function $\phi_{1}(x / \sqrt{\zeta})$, we can expand its 2-point function as

$$
\left\langle\phi_{1}(t) \mid \phi_{1}(0)\right\rangle \sim a\left(\frac{\zeta}{R t}\right)^{2 d}+\text { higher order terms in } \frac{\zeta}{R t},
$$

where $d$ is the dimension of $\phi_{1}$ in the quantum mechanics (which, as described above, is proportional to $T$ ). We then define a renormalized operator by $\phi_{1} \rightarrow(\zeta / R)^{-d} a^{-1 / 2} \phi_{1}$. This wave function renormalization is performed such that the coefficient of the identity operator in the 2-point function is exactly $\frac{1}{t^{2 d}}$. The terms of higher order in $\frac{1}{t}$ will have powers of $\zeta$ in them, and are, therefore, cut-off artifacts.

For any choice of generic wave functions $\phi_{1}$ and $\phi_{2}$ with the same quantum numbers (under global symmetries), the large- $t$ behavior of the 2-point function is dominated by the conformal state of lowest dimension with the same quantum numbers, i.e, the two point functions $\left\langle\phi_{i}(t) \mid \phi_{i}(0)\right\rangle, i=1,2$ decay (for large $t$ ) with the same power of $t$. From these two functions, however, we can extract a state that corresponds to an operator with higher

interested in large values of $C$ (or $H$ ) in Planck units, when the low-energy description is no longer valid.

${ }^{12} \mathrm{~A}$ similar normalization of wave functions to cancel the dependence on a cutoff which is a FI term appears in [40]. 
dimension by taking a linear combination $\phi_{3}$ such that the large- $t$ behavior of $\left\langle\phi_{3}(t) \mid \phi_{3}(0)\right\rangle$ now falls off with a higher power of $\frac{1}{t}$. One again needs to rescale this operator such that the leading contribution does not depend on $\zeta$, and interpret all remaining $\zeta$ dependence as a cut-off artifact. $\phi_{3}$ can now serve as a representative for the next-to-leading operator with the same quantum numbers, as far as the large- $t$ behavior is concerned.

Next, let us go over to the 3 -point functions. Taking some 3 operators $\psi_{1,2,3}$ and calculating their 3-point function, one obtains an expansion (before the wave function renormalization) for $\left\langle\psi_{1}\left(t_{1}\right) \psi_{2}\left(t_{2}\right) \psi_{3}(0)\right\rangle$ of the form

$$
c\left(\frac{\zeta}{R t_{1}}\right)^{d_{1}+d_{3}-d_{2}}\left(\frac{\zeta}{R t_{2}}\right)^{d_{2}+d_{3}-d_{1}}\left(\frac{\zeta}{R\left(t_{1}-t_{2}\right)}\right)^{d_{1}+d_{2}-d_{3}}+\cdots,
$$

where $(\cdots)$ denotes higher order terms in $\zeta / R t$. After the wave function renormalization, the $\zeta$ dependence disappears from the leading term, which becomes the 3-point function of the operators. Subleading terms are, as before, cut-off artifacts.

To conclude, the definition of the states in the conformal field theory, i.e. when $\zeta=0$, is as the Hilbert space of states on the resolved target space $\tilde{\mathcal{M}}_{N, k}$, modulo a relation. The relation is that two such elements in the Hilbert space are equivalent if the leading $\frac{1}{t}$ dependence of all their correlation functions are the same. After this identification, we can translate the finite- $\zeta$ wave functions to the conformal states by the procedure described above.

An example of this is the following. Let us consider the real axis, and the evolution of functions by the heat equation. Upon Fourier transform, the long time behavior of the wave function is governed by the Taylor expansion of the Fourier transform around the origin $P=0, \hat{f}(p)=a_{0}+a_{1} p+\frac{1}{2} a_{2} p^{2}+$ $\ldots$. The equivalence class of the state with the least dimension contains all the states with $a_{0} \neq 0$, the equivalence class of the state with the next-toleading dimension is given by functions with $a_{0}=0, a_{1} \neq 0$, etc.

We cannot do such a computation explicitly in our case, as the space is quite complicated. However, the general structure will be the same. The Fourier transform will be replaced by the spectral representation of the Schrödinger equation. The relevant components of the spectrum will be either normalizable discrete zeros or a continuum component that goes down to zero. Two functions will give the same state in the conformal limit if their behavior at eigenvalues close to zero is the same.

We will not analyze the general equivalence relation any further, as we will be interested in a special class of operators and states that are easier to control, which correspond to chiral primary fields. In order to analyze these, we need to understand better the supersymmetries of the resolved model. 


\subsection{Supersymmetries and Forms in Quantum Mechanical Sigma Models}

After the resolution of the singularities, we have a quantum mechanical sigma model with 8 supercharges on a smooth (though non-compact) manifold. The theory after the resolution is no longer superconformal. In this subsection we describe a convenient description of the fermionic variables and supersymmetries of such models in terms of forms on the target space.

Let us begin by assuming that we have a quantum mechanical supersymmetric sigma model with two supercharges (or one complex supercharge $Q$ ) on a manifold $\mathcal{M}$. Then, it is well known [41] that the states of the sigma model may be identified with forms on $\mathcal{M}$, and one of the supercharges may be identified with the exterior derivative operator $d$ acting on these forms. The SUSY algebra acts as $\left[Q, x^{i}\right]=\theta^{i}$ (where $\theta^{i}$ is a complex fermion), and the fermionic zero modes obey $\left\{\left(\theta^{i}\right)^{\dagger}, \theta^{j}\right\}=g^{i j}$ where $g$ is the metric on the space. We can choose the vacuum such that $\theta_{j}|0\rangle=0$, and then we can identify the fermion creation operators $\left(\theta^{i}\right)^{\dagger}$ with forms $d x^{i}$, and the annihilation operators $\theta^{i}$ with $* d x^{i} *$. This allows us to translate any state in the quantum mechanics into a form on the target space. The SUSY generator $Q \sim \theta_{i}^{\dagger} \partial_{i}+\Gamma_{j k}^{i}(x) \theta_{i} \theta_{j}^{\dagger} \theta_{k}^{\dagger}$ may then be identified with the exterior derivative operator $d$ on the space (the other SUSY generator corresponds to $d^{\dagger}=* d *$ ). Thus, the space of states which are annihilated by $Q$ is translated into the space of closed forms on the target space $\mathcal{M}$.

The $\mathcal{N}=2$ SUSY algebra includes a $U(1)_{R}$ symmetry, which $Q$ and all the fermionic fields are charged under. The identification above means that the $U(1)_{R}$ charge of a state is equal to the degree of the corresponding form, up to a constant shift (the $U(1)_{R}$ charge of a $p$-form state is $p-\frac{1}{2} \operatorname{dim}(\mathcal{M})$ ).

In our case we actually have 8 (real) supercharges, and the R symmetry is $S U(2)_{R} \times \operatorname{Spin}(5)$. The $S U(2)_{R}$ is broken by $\zeta$ to $U(1)_{\zeta}$. Choosing an $\mathcal{N}=2$ subalgebra includes a choice of a $U(1)_{R}$ symmetry in $U(1)_{\zeta} \times \operatorname{Spin}(5)$. Since we want a $U(1)_{R}$ symmetry that does not act on the bosonic coordinates, we should choose $U(1)_{R} \subset \operatorname{Spin}(5)$, and it seems that the unique consistent choice of a $U(1)_{R}$ is to use the $U(1)$ which appears in the maximal subgroup of $\operatorname{Spin}(5) \simeq S p(2)$ which is $S U(2) \times U(1)$. In particular, the 4 of $\operatorname{Spin}(5)$ decomposes as $\mathbf{2}_{\mathbf{1}}+\mathbf{2}_{-\mathbf{1}}$, so with this choice of $U(1)_{R}$ we are assured that all the fermionic coordinates will have charge \pm 1 , as required for the realization of the SUSY algebra described above. This choice is also required to ensure that the fermions transform as the tangent bundle of the manifold, as needed for their identification with forms.

After this choice of an $\mathcal{N}=2$ subalgebra of the quantum mechanical supersymmetry, one of the $Q$ operators with charge $(+1)$ under the $U(1)_{R}$ symmetry will be the $\mathcal{N}=2$ supersymmetry generator, which may be identified with the exterior derivative operator $d$ (its conjugate is identified with 
$\left.d^{\dagger}\right)$. The other components of $Q$ correspond to other differential operators on $\mathcal{M}$, of degrees \pm 1 .

\section{Representations of the Conformal Algebra and Correlators in DLCQ}

\subsection{Representations of the Superconformal Algebra in DLCQ}

The interesting representations of the conformal group for physical applications are representations containing a primary field $\Phi$. The action of the conformal generators on such a field is given by

$$
\begin{aligned}
{\left[P_{\mu}, \Phi(x)\right] } & =i \partial_{\mu} \Phi(x) \\
{\left[M_{\mu \nu}, \Phi(x)\right] } & =\left[i\left(x_{\mu} \partial_{\nu}-x_{\nu} \partial_{\mu}\right)+\Sigma_{\mu \nu}\right] \Phi(x) \\
{[D, \Phi(x)] } & =i\left(d+x^{\nu} \partial_{\nu}\right) \Phi(x) \\
{\left[K_{\mu}, \Phi(x)\right] } & =\left[-i\left(x^{2} \partial_{\mu}-2 x_{\mu} x^{\nu} \partial_{\nu}-2 x_{\mu} d\right)+2 x^{\nu} \Sigma_{\mu \nu}\right] \Phi(x)
\end{aligned}
$$

and, in particular, $\left[K_{\mu}, \Phi(0)\right]=0 . d$ is called the dimension of the field $\Phi$, and $\Sigma_{\mu \nu}$ are the usual spin representations of the Lorentz group.

In the usual construction of representations of the conformal group, $D$ is diagonalized. The primary field (at $x=0$ ) is annihilated by the generators $K_{\mu}$ of the conformal group which lower the dimension $d$, while the action of the other conformal generators on the primary field gives us the full conformal block. In particular, the fields fall into representations of the Lorentz group and of any other global symmetry, and the generators of these groups commute with $D$. For the superconformal group the story is similar, with the $S$ generators of the superconformal group annihilating primary states, while the supersymmetry generators $Q$ raise the value of $d$ and generate the conformal block.

In the discrete light-cone quantization, the representation structure is different since we are looking only at a sector with a particular eigenvalue of $P_{-}$. In particular, this sector may include a particular momentum mode of all the fields of the conformal block described above. Since, as discussed above, $D$ does not commute with $P_{-}$, we cannot diagonalize $D$, and instead we diagonalize $T=D-M_{01}$. We will call the eigenvalue of $T$ (divided by i) the "DLCQ dimension" of a field, and denote it by $t_{0}$. We also do not see full representations of the Lorentz group, but only of the part of the group that commutes with $P_{-}$, as described above. In particular, for the $(2,0)$ superconformal theories in six dimensions, we will sort the states according to the eigenvalue of $T$ and the $S U(2)_{R} \times S U(2)_{L} \times \operatorname{Spin}(5)$ representation. 
As before, we will look for states which are annihilated by the generators of the conformal group which lower the "DLCQ dimension" of a field. These generators are $K_{-}, V_{i}$ and $S$. Given such a state, we can form its full conformal block by acting on it with the other superconformal generators. The generators $\tilde{Q}$ and the $S U(2)_{R} \times S U(2)_{L} \times \operatorname{Spin}(5)$ charges commute with $T$, and generate states with the same "DLCQ dimension." The other operators $Q, P_{i}$ and $H$, raise the "DLCQ dimension" of a field, and generate various descendants of a primary field.

As an example let us analyze the free tensor multiplet. It contains five scalar fields, in the $(\mathbf{1}, \mathbf{5})$ representation of $S O(5,1) \times \operatorname{Spin}(5)$, one fermion field in the $(4,4)$ representation and one self-dual 3 -form field strength in the $(\mathbf{1 0}, \mathbf{1})$ representation. Since these are all free fields, their dimensions are $d=2,5 / 2$ and 3 respectively. The full representation of the superconformal algebra is completed by additional operations with $Q$ and $P$ on these fields.

In DLCQ, we can easily compute the $T$-eigenvalues and $S U(2)_{R} \times S U(2)_{L}$ $\times \operatorname{Spin}(5)$ representations of all of these fields. The 5 scalars all have a "DLCQ dimension" $t_{0}=2$, and are in the $(\mathbf{1}, \mathbf{1}, \mathbf{5})$ representation. The fermions split according to their $M_{01} \sim \Gamma^{0} \Gamma^{1}$ eigenvalue, into a $(\mathbf{1}, \mathbf{2}, \mathbf{4})$ representation with $t_{0}=2$ and a $(\mathbf{2}, \mathbf{1}, 4)$ representation with $t_{0}=3$. The Dirac equation for a free fermion of momentum $P_{-}=N / R$ allows us to express the $t_{0}=3$ components as derivatives (descendants) of the $t_{0}=2$ components. The tensor field splits into a $(\mathbf{1}, \mathbf{3}, \mathbf{1})$ with $t_{0}=2$, a $(\mathbf{2}, \mathbf{2}, \mathbf{1})$ with $t_{0}=3$ and a $(\mathbf{3}, \mathbf{1}, \mathbf{1})$ with $t_{0}=4$. The $t_{0}=3$ and $t_{0}=4$ states are again descendants, in the DLCQ, of the $t_{0}=2$ states. The $t_{0}=2$ states of the tensor multiplet form an irreducible representation of the Clifford algebra of the $\tilde{Q}$ operators. Generally, to generate all the states we will need to also use the $S U(2)_{R} \times S U(2)_{L} \times S p i n(5)$ charges.

A special class of primary fields is the class of chiral primary fields. These are representations of the superconformal group that contain null states. We will discuss here only chiral primary fields for which some combination of supercharges $Q$ acting on the primary states in the representation (states with the minimal dimension) vanishes. We will further restrict ourselves to representations whose primary fields are Lorentz scalars. The superconformal algebra may be used to derive a bound on the dimension of primary fields, given their R-symmetry representation, which is exactly saturated for chiral primary fields. The free tensor multiplet described above is the simplest case of a chiral primary field.

We will not derive here the general equation for the dimension of chiral primary fields $[42,43]$, but only a special case that will be useful in the following. The $(2,0)$ superconformal algebra in six dimensions contains as a subalgebra the $(1,0)$ superconformal algebra. The R-symmetry of the $(1,0)$ algebra is $S U(2)$, and this $S U(2)$ may be chosen to be either of the two 
$S U(2)$ factors in the maximal subgroup $S U(2) \times S U(2) \subset S p i n(5)_{R}$ of the R-symmetry group of the $(2,0)$ algebra. The $(1,0)$ superconformal algebra may be used to show that the bound on the dimension of a primary scalar field in the $2 j+1$ dimensional representation of the $S U(2)$ R-symmetry is $d \geq 4 j$, and it is saturated only for chiral primary fields of the $(1,0)$ superconformal group. The $\mathbf{5}$ representation of $\operatorname{Spin}(5)$ decomposes into $(\mathbf{2}, \mathbf{2})+(\mathbf{1}, \mathbf{1})$ of $S U(2) \times S U(2)$, so we find that the bound on a scalar field in the 5 representation is at least $d \geq 2$ (since obviously a primary field of the $(2,0)$ algebra is also a primary field of the $(1,0)$ theory). In this case this bound is actually the maximal one, as the example of the free tensor multiplet demonstrates. Similarly, one can easily show that the dimension of a primary scalar field in the $n$-th symmetric product of 5 's obeys $d \geq 2 n$, since its decomposition into $S U(2) \times S U(2)$ representations includes $(\mathbf{n}+\mathbf{1}, \mathbf{n}+\mathbf{1})$, and a field which saturates this bound is necessarily a chiral primary field (since it is a chiral primary field of the $(1,0)$ subalgebra).

\subsection{2-point Functions of Primary Operators in DLCQ}

The correlation functions of scalar primary fields are strongly constrained by the conformal algebra. Their 2-point functions are of the form

$$
\left\langle\Phi_{i}^{\dagger}(x) \Phi_{i}(0)\right\rangle=\frac{1}{\left(x^{2}\right)^{d_{i}}},
$$

and their 3-point functions are of the form

$$
\begin{aligned}
\left\langle\Phi_{i}\left(x_{1}\right)\right. & \left.\Phi_{j}\left(x_{2}\right) \Phi_{k}\left(x_{3}\right)\right\rangle \\
& =\frac{c_{i j k}}{\left[\left(x_{12}\right)^{2}\right]^{\left(d_{i}+d_{j}-d_{k}\right) / 2}\left[\left(x_{13}\right)^{2}\right]^{\left(d_{i}+d_{k}-d_{j}\right) / 2}\left[\left(x_{23}\right)^{2}\right]^{\left(d_{k}+d_{j}-d_{i}\right) / 2}},
\end{aligned}
$$

where $d_{i}$ is the dimension of $\Phi_{i}$ and $x_{i j}=x_{i}-x_{j}$.

In the DLCQ, we are not studying the operators $\Phi$, but rather their momentum modes

$$
\Phi_{N}\left(x^{i}, x^{+}\right)=\int_{0}^{2 \pi R} d x^{-} e^{-i N x^{-} / R} \Phi\left(x^{i}, x^{+}, x^{-}\right) .
$$

We are interested in computing things like the 2-point function of these momentum modes, $f_{N}\left(x^{i}, x^{+}\right)=\left\langle\Phi_{N}^{\dagger}\left(x^{i}, x^{+}\right) \Phi_{N}(0)\right\rangle$. The remaining conformal symmetry (which commutes with $P_{-}$) is still enough to determine this up to a constant. The rotation symmetries require $f_{N}\left(x^{i}, x^{+}\right)=f_{N}\left(\left(x^{i}\right)^{2}, x^{+}\right)$. The action of $T$ then requires $f_{N}\left(x^{i}, x^{+}\right)=\frac{1}{\left(x^{+}\right)^{d}} \hat{f}_{N}\left(\left(x^{i}\right)^{2} / x^{+}\right)$. Since the operator (35) is an eigenstate of $T$, and $T$ is the same algebra element for every $N, d$ does not depend on $N$. Then, the action of $K_{-}$uniquely determines

$$
\left\langle\Phi_{N}^{\dagger}\left(x^{i}, x^{+}\right) \Phi_{N}(0)\right\rangle \propto \frac{1}{\left(x^{+}\right)^{d}} e^{-i N\left(x^{i}\right)^{2} / 4 R x^{+}} .
$$


In particular, we can determine the dimension of primary operators in the DLCQ without having to go to large values of $N$. For higher $n$-point functions we may have to go to large $N$ to get exact results in the space-time theory.

\section{Primary States and Correlation Functions from the Quantum Mechanics}

The remaining obstacle to calculating dimensions and correlation functions is the precise identification between states in space-time and states in the quantum mechanics. As explained in section 4, states of the conformal field theory in space-time (without the cutoff) are realized in the quantum mechanics as equivalence classes in the Hilbert space of the sigma model on the resolved space $\tilde{\mathcal{M}}_{N, k}$. We defined these equivalence classes using the long time evolution of wave functions, but actually computing the equivalences is quite complicated, and we will do it for some simple examples below.

\subsection{Identifying Chiral Primary Operators in the Quantum Mechanics}

As described above, any primary operator in the quantum mechanics must be annihilated by $K_{-}$. Chiral primary operators (of the space-time theory) are also annihilated by some of the spacetime SUSY generators (in addition to being annihilated by $K_{-}$). As described above, the states that are annihilated by one particular $Q$, which generates (together with $Q^{\dagger}$ ) an $\mathcal{N}=2$ subalgebra, correspond to closed forms on the target space. We will focus here on this particular type of chiral primary states.

For our purposes, we are interested in states that are annihilated by $Q$ and are also concentrated at the origin of the moduli space. Since there is an obvious scaling symmetry of the target space (for $\zeta=0$ ), we can scale any state which has compact support (namely, it vanishes sufficiently fast on the boundary at infinity of the manifold) so that it is concentrated at the origin of moduli space. Thus, the chiral primary operators of an $\mathcal{N}=2$ superconformal sigma model are given by the cohomology with compact support (sometimes denoted by $H\left(\mathcal{M}_{N, k}, \partial \mathcal{M}_{N, k}\right)$ ) of the target space (which, in our case, is the instanton moduli space $\mathcal{M}_{N, k}$ ). Note that we can also use the $\mathcal{N}=2$ quantum mechanical superconformal algebra to learn about the dimensions of chiral primary operators. The $\mathcal{N}=2$ supersymmetry algebra contains a $U(1)_{R}$ symmetry, and the superconformal algebra gives a bound on the dimensions of primary operators, $T \geq|R|$, with equality only for chiral primary operators (we normalize the R-charge of $Q$ to be one, and $T \sim 2 D_{Q M}$ as described above). 
The justification for identifying states differing by an exact form is the following. Adding an exact form to a state certainly changes the state, and also, for instance, its 2-point functions. This can be identified with adding the commutator of $Q$ with some operator to our primary state. However, such an exact form will always have a higher "DLCQ dimension," because it will not saturate the bound relating the dimension to the R-charge. Thus, its correlation functions will decay faster in time, and the long-time behavior will not depend on such additions of exact forms. The large-time correlation functions depend only on the cohomology, which is the reason why we can identify the cohomology with (a subgroup of) the chiral primary states.

In our case we actually have 8 supersymmetry generators, and we are looking for states that are annihilated by some combination of the supersymmetry generators. The simplest way to look for chiral primary operators is to take an $\mathcal{N}=2$ subalgebra of the $\mathcal{N}=8$ SUSY algebra. Given such a choice of an $\mathcal{N}=2$ SUSY generator $Q$, the states annihilated by $Q$ will correspond, as described above, to the cohomology with compact support of $\mathcal{M}_{N, k}$. Obviously, a primary state that is annihilated by the $\mathcal{N}=2$ SUSY generator corresponds to a chiral primary operator, since this generator is a combination of the $\mathcal{N}=8$ SUSY generators.

However, the converse is not necessarily true. There could be representations which are chiral primaries of the $\mathcal{N}=8$ algebra but which do not include any chiral primaries of the $\mathcal{N}=2$ subalgebra. A chiral primary of the $\mathcal{N}=8$ superconformal algebra, in some representation of $S U(2)_{R} \times \operatorname{Spin}(5)$ and with some "DLCQ dimension" $t_{0}$, includes states of different $U(1)_{R}$ charge ${ }^{13}$. If the representation includes a state with $R=t_{0}$, this state will be a chiral representation also of the $\mathcal{N}=2$ subalgebra, and we will find it in the cohomology. On the other hand, if the highest $U(1)_{R}$ charge appearing in the decomposition is lower than this bound, the decomposition of this $\mathcal{N}=8$ representation into $\mathcal{N}=2$ representations will not include any chiral primary operators, so we will not find a representative of this state in the cohomology. For scalars in space-time, which are hence $S U(2)_{R}$ singlets, it is easy to check that the only Spin(5) representations which obey this condition are the totally symmetric (traceless in each pair of indices) products of 5's. Thus, only chiral primary operators in totally symmetric (traceless) representations will correspond to the cohomology with compact support of $\mathcal{M}_{N, k}$. Other chiral primary operators will be annihilated by other linear combinations of $Q$ 's, and we leave their analysis to future work [22].

Note that the derivation above proves that the bound on the dimension of primary operators in the quantum mechanics which are singlets of $S U(2)_{R}$ and which are in the $n$-symmetric traceless representation of $\operatorname{Spin}(5)$ is $T \geq$

\footnotetext{
${ }^{13} t_{0}$ is determined in terms of the $S U(2)_{R} \times \operatorname{Spin}(5)$ representation by the $\mathcal{N}=8$ superconformal algebra.
} 
$2 n$. Using the relation to the space-time theory, it also shows that the bound on the dimension of scalar primary operators in $(2,0)$ six dimensional SCFTs in the same representation of $\operatorname{Spin}(5)_{R}$ is $d \geq 2 n$, with equality exactly for chiral primary operators, as we found above by using a $(1,0)$ subalgebra. This is a special case of the general correspondence between R-symmetry representations and dimensions of primary operators.

\subsection{Computation of the Cohomology}

Now that we have resolved the singularities, the computation of the cohomology with compact support of the resolved space $\tilde{\mathcal{M}}_{N, k}$ becomes a welldefined mathematical problem. An algorithm for computing the homology using Morse theory was given in [33], and an explicit formula appears in [44] (a different proof will appear in [45]). After translating the formula from homology to cohomology with compact support by the usual duality, it states that a generating function for the dimension $a_{p, N}$ of $H^{p}\left(\tilde{\mathcal{M}}_{N, k}\right)^{14}$ is

$$
\prod_{i=1}^{\infty} \prod_{j=1}^{k} \frac{1}{1-t^{2 j} q^{i}}=\sum_{N, p} a_{p+2 N k, N} t^{p} q^{N} .
$$

Let us begin by interpreting this result for $N=1$. In this case, we find a one dimensional cohomology for $p=2 k+2,2 k+4, \cdots, 4 k$. Using the relation between the $R$-charge in the $\mathcal{N}=2$ superconformal algebra and the degree of the form (which is $R=p-\frac{1}{2} \operatorname{dim}\left(\mathcal{M}_{N, k}\right)=p-2 N k$ ), this implies the existence of $\mathcal{N}=2$ superconformal chiral primary states with $R=2,4, \cdots, 2 k$. As described above, this implies the existence of states with $t_{0}=2,4, \cdots, 2 k$, where the state with $t_{0}=2 n$ is in a $\operatorname{Spin}(5)$ representation corresponding to the $n$ 'th symmetric (traceless) product of 5's. Note that an important consistency check on this procedure is that (37) never predicts states with negative dimension (or even states whose dimension is smaller than 2).

The states we found for $N=1$ have a natural interpretation as the coordinates on the moduli space of the space-time theory, which is $\mathbb{R}^{5 k} / S_{k}$. At a generic point on the moduli space, the low-energy field content is $k$ tensor multiplets, each including 5 scalars $\phi_{a}^{i}, i=1, \cdots, 5, a=1, \cdots, k$, which are the natural local coordinates on the moduli space. It is natural to look at (globally well-defined) functions on the moduli space which are the symmetric products of these scalar fields. The symmetric products of more than $k$ fields are determined by the symmetric products with less fields ${ }^{15}$.

\footnotetext{
${ }^{14} H^{p}\left(\tilde{\mathcal{M}}_{N, k}\right)$, the space of $p$-forms in the cohomology, is isomorphic to $H_{4 N k-p}\left(\tilde{\mathcal{M}}_{N, k}\right)$, which is the space of $(4 N k-p)$-cycles in the homology.

${ }^{15}$ Note that even the symmetric products with $k$ fields or less are not completely independent.
} 
We find that for $N=1$ we have exactly one state corresponding to the momentum one component of each such symmetric product (of $k$ fields or less), and no other state in a symmetric $\operatorname{Spin}(5)$ representation. We will denote the operator in the $l$-symmetric product of 5 's by $u_{l}$. A special case is $u_{1}$ which has dimension 2 , and is, therefore, a free field (this can be proven by using the superconformal algebra). This is exactly the free decoupled tensor multiplet, whose scalars correspond to the center of mass position of the $k$-branes.

Given this field content, we would expect to have states for arbitrary $N$ of the form $\prod_{i}\left(u_{l_{i}}\right)_{N_{i}}$ where $\left(u_{l}\right)_{n}$ is the $n$-momentum mode of $u_{l}, 1 \leq l_{i} \leq k$, the $\operatorname{Spin}(5)$ representations are multiplied symmetrically ${ }^{16}$, and $\sum_{i} N_{i}=N$. It is easy to compute the contribution of these states to the generating function (37), with each such state appearing at a value of $p$ corresponding to a "DLCQ dimension" $t_{0}=\sum_{i} 2 l_{i}$, and we find that it is exactly equal to (37). Thus, it seems that all chiral primary states in symmetric $\operatorname{Spin}(5)$ representations are of this form. Specifically, the only chiral primary operators in symmetric (traceless) representations of $\operatorname{Spin}(5)$ are products of the $u_{l}$ fields, and a product of $j u_{l}$ fields appears for the first time at momentum $N=j$. Note that apriori we do not know at which momentum a particular field will first appear. Since our computation of the spectrum of chiral primary operators in symmetric representations is valid for all values of $N$, it is valid also in the large $N$ limit, which corresponds to a light-cone description of the uncompactified six dimensional theory.

For $k=1$ this result was described already in [18], from a slightly different point of view. In this case the theory is free, and the states we find here correspond exactly to the chiral primary states of the conformal theory of a free tensor field. In particular, from the analysis of the free field theory we find that all the chiral primary states are in totally symmetric (traceless) representations (since anti-symmetric products vanish), so our construction gives us all the chiral primary states in this case.

\section{$7 \quad$ Some Simple Examples}

\subsection{States and Correlation Functions for $N=1 k=1$}

In this section we will give some concrete examples of the general construction of chiral primary states described in the previous section. The simplest

\footnotetext{
${ }^{16}$ Note that there are no singularities in symmetric products of chiral primary fields in symmetric representations, since the dimension of the product is equal to the sum of the dimensions of the fields. Generally, this would not be true, so there is no chiral ring for $(2,0)$ superconformal theories.
} 
example is $N=1 k=1$. The theories with $k=1$ (corresponding to $U(1)$ instantons, which are pointlike) are always expected to be free. The case $N=1$ is particularly simple, since the instanton moduli space in this case is just $\mathbb{R}^{4}$. In this case the deformation by $\zeta$ has no effect, and we can do all our computations without it. Still, it is worthwhile to analyze this case in some detail, both because it is the simplest case, and because the moduli space always includes a decoupled $\mathbb{R}^{4}$ factor, and the behavior of the states in this $\mathbb{R}^{4}$ component will generally be very similar to the behavior for $N=1 k=1$.

Using the formula (37), we find that in this case there is only one form in the cohomology with compact support, with degree 4 . It is dual to the only element in the homology, which is the point. An element of this cohomology may be chosen to be $\hat{f}=f\left(x^{1}, x^{2}, x^{3}, x^{4}\right) d x^{1} \wedge d x^{2} \wedge d x^{3} \wedge d x^{4}$ for any function $f$ which has compact support and obeys $\int_{\mathbb{R}^{4}} \hat{f} \neq 0$. Then, it is easy to explicitly check that this state is in the cohomology with compact support, namely that $d \hat{f}=0$ and that there is no $\hat{g}$ with compact support such that $\hat{f}=d \hat{g}$. As described above, to get the actual chiral primary state we have to scale this state to the origin, so that it is annihilated by $K_{-}$ (which in this case is proportional to $|x|^{2}$ ). The resulting state is thus

$$
F=\prod_{i} \delta\left(x^{i}\right) d x^{1} \wedge d x^{2} \wedge d x^{3} \wedge d x^{4},
$$

up to a normalization constant which we will determine by computing the 2 -point function below ${ }^{17}$. According to the general arguments above, this state represents one of the scalar fields of the free tensor multiplet $u_{1}$ with one unit of longitudinal momentum.

It is very simple in this case to find the other states with $t_{0}=2$ in the free tensor multiplet, since they may be generated by acting with $\tilde{Q}$ on the state we found. In this case the $\tilde{Q}$ are just the fermionic partners of the $\mathbb{R}^{4}$ coordinates, which in the form language correspond to $d x^{i}$ and $* d x^{i} *$ (in fact, this is true for all values of $N$ and $k$ ). Acting with these operators on the state we found, we find 16 states, of the form $\prod_{i} \delta\left(x^{i}\right)$ times any number of $d x^{i}$ s.

The $\operatorname{Spin}(5)$ currents act only on the fermions, and not on the coordinates of the moduli space. As described above, we have already chosen a $U(1)_{R} \subset$ $\operatorname{Spin}(5)$ such that its charge corresponds (up to a shift by $2 N k$ ) to the degree of a form. There is an $S U(2) \subset \operatorname{Spin}(5)$ which commutes with this $U(1)_{R}$, and the decomposition of the adjoint 10 representation of $\operatorname{Spin}(5)$ into $S U(2) \times U(1)_{R}$ representations is $\mathbf{3}_{\mathbf{2}}+\mathbf{1}_{\mathbf{0}}+\mathbf{3}_{\mathbf{0}}+\mathbf{3}_{-\mathbf{2}}$. The $\mathbf{1}_{\mathbf{0}}$ component

\footnotetext{
${ }^{17}$ Note that here we choose a different convention than in $\S 4.4$, in which the wave function depends on $x$ and not on $x / \sqrt{\zeta}$. Of course, after the wave function normalization we will get the same results.
} 
of the $\operatorname{Spin}(5)$ charge is just the $U(1)_{R}$ generator, which may be written as $g_{i j} d x^{i} * d x^{j} *$. The $\mathbf{3}_{0}$ components generate the $S U(2) \subset \operatorname{Spin}(5)$, and may be written as $g_{i j}\left(J^{a}\right)_{k}^{j} d x^{i} * d x^{k} *$, where $J^{a}$ are the three covariantly constant complex structures of the manifold. The $\mathbf{3}_{\mathbf{2}}$ components are represented as a multiplication by the three Kähler forms, each of which is a $(1,1)$ form with respect to one of the 3 covariantly constant complex structures of the manifold. In our example of $N=1 k=1$, they may be chosen to be $d x^{1} \wedge d x^{2}+d x^{3} \wedge d x^{4}, d x^{1} \wedge d x^{3}-d x^{2} \wedge d x^{4}$ and $d x^{1} \wedge d x^{4}+d x^{2} \wedge d x^{3}$. The $\mathbf{3}_{-2}$ generators are the conjugates of these (by the star operation).

The $S U(2)_{R}$ and $S U(2)_{L}$ currents are more complicated, since they act also on the bosonic coordinates, and they correspond to geometrical symmetries of the manifold. Obviously they commute with $U(1)_{R}$, so they do not change the degree of a form. $S U(2)_{R}$ acts only on the bosonic coordinates and not on the fermionic coordinates, and in the simple case of $N=1$ $k=1$, it is a subgroup of the $S O(4)$ rotation symmetry of $\mathbb{R}^{4}$, generated by $\left(x^{i} \frac{\partial}{\partial x^{j}}-x^{j} \frac{\partial}{\partial x^{i}}\right) . S U(2)_{L}$ is slightly more complicated, since it acts also on the fermions - its generators are similarly a subgroup of $S O(4)$ but with an action also on the forms and not only on their coefficient function.

Using these symmetry generators, it is easy to check that the 16 states we found above are in the $(\mathbf{5}, \mathbf{1})+(\mathbf{4}, \mathbf{2})+(\mathbf{1}, \mathbf{3})$ representation of $\operatorname{Spin}(5) \times$ $S U(2)_{L}$, and singlets of $S U(2)_{R}$. This is also clear since the $\tilde{Q}$ generators are in the $(\mathbf{1}, \mathbf{2}, \mathbf{4})$ of $S U(2)_{R} \times S U(2)_{L} \times \operatorname{Spin}(5)$, and the 16 states are generated by zero modes in this representation.

Other states in the superconformal representation of the free tensor multiplet may be generated by acting with the other space-time superconformal generators on the state we found. In this case the only operators which do not vanish when acting on $F$ of (38) are $P_{i}, H$ and $Q$, leading to various derivatives of the delta function (all the states we describe here are eigenstates of $T$ ). The operator $P_{i}$ acts by shifting $x^{i}$. Thus, it is clear that an insertion of an operator at a position $x_{0}^{i}$ is described by exchanging $\delta(x)$ above with $\delta\left(x-x_{0}\right)$.

Next, let us compute the 2-point functions of the chiral primary states that we found. For instance, for the state $F$ described above, the 2-point function will be given by $\int_{\mathbb{R}^{4}}\left(e^{i H t} F\left(x_{1}\right)\right) \wedge * F\left(x_{2}\right)$, or explicitly

$$
\left\langle F^{\dagger}\left(x_{1}, t\right) F\left(x_{2}, 0\right)\right\rangle=\int d^{4} x\left[e^{i R t\left(\partial / \partial x^{i}\right)^{2}} \delta^{4}\left(x-x_{1}\right)\right] \delta^{4}\left(x-x_{2}\right),
$$

where we denote $\delta^{4}(x)=\prod_{i=1}^{4} \delta\left(x^{i}\right)$. The time evolution in this case is very simple, and we find

$$
\left\langle F^{\dagger}\left(x_{1}, t\right) F\left(x_{2}, 0\right)\right\rangle=-\frac{1}{16 \pi^{2} R^{2} t^{2}} e^{-i\left(x_{1}-x_{2}\right)^{2} / 4 R t} .
$$


Up to normalization constants, this is exactly the expected result for a chiral primary operator of dimension 2 , as described in section 5 . The other 16 states described above all have the same 2-point functions with their conjugate states, while all off-diagonal 2-point functions vanish.

\subsection{States and Correlation Functions for $N=1, k=2$ and $N=2, k=1$}

The next simplest example is either $N=1, k=2$ or $N=2, k=1$. In both of these cases the moduli space is of the form $\mathbb{R}^{4} \times \mathbb{R}^{4} / \mathbb{Z}_{2}$, which we will parametrize by coordinates $\tilde{x}^{i}$ and $v^{i}$ respectively, though the interpretation of the states is quite different in the two cases ${ }^{18}$. In either case, the parameter $\zeta$ is the usual blowup parameter for the $\mathbb{R}^{4} / \mathbb{Z}_{2}$ singularity.

The formula (37) predicts two cohomology elements, one which is an 8form and one which is a 6 -form. We will denote the corresponding states by $F_{8}$ and $F_{6}$. The 8 -form is similar to the 4 -form described in the previous subsection, and we may construct the corresponding chiral primary state even in the limit $\zeta \rightarrow 0$. The corresponding form is $\delta^{4}(\tilde{x}) \delta^{4}(v) \prod_{i} d \tilde{x}^{i} \prod_{j} d v^{j}$. Again, we can translate this state in space using the $P_{i}$, giving $\delta^{4}(\tilde{x}-$ $\left.x_{0}\right) \delta^{4}(v) \prod_{i} d \tilde{x}^{i} \prod_{j} d v^{j}$. We can also translate the state in time using the evolution operator $H$ in the quantum mechanics, which is simply given by $H=\frac{R}{2}\left(\partial_{\tilde{x}^{i}}^{2}+\partial_{v^{j}}^{2}\right)$. This turns the state into

$$
F_{8}\left(x_{0}, t\right) \simeq \frac{1}{t^{4}} e^{-\left(\left(\tilde{x}^{i}-x_{0}^{i}\right)^{2}+\left(v^{j}\right)^{2}\right) / 2 i R t} \prod_{i} d \tilde{x}^{i} \prod_{j} d v^{j},
$$

up to ( $R$-dependent) constants which we will ignore throughout.

It is again easy to compute 2-point functions of this state, and we find

$$
\begin{aligned}
& \left\langle F_{8}^{\dagger}\left(x_{1}, t_{1}\right) F_{8}\left(x_{2}, t_{2}\right)\right\rangle \\
& \quad \propto \int d^{4} \tilde{x} d^{4} v \frac{1}{t_{1}^{4}} \frac{1}{t_{2}^{4}} e^{\left(\left(\tilde{x}^{i}-x_{1}^{i}\right)^{2}+\left(v^{i}\right)^{2}\right) / 2 i R t_{1}} e^{-\left(\left(\tilde{x}^{i}-x_{2}^{i}\right)^{2}+\left(v^{i}\right)^{2}\right) / 2 i R t_{2}} \\
& \quad \propto \frac{1}{\left(t_{1}-t_{2}\right)^{4}} e^{-i\left(x_{1}^{i}-x_{2}^{i}\right)^{2} / 2 R\left(t_{1}-t_{2}\right)} .
\end{aligned}
$$

Up to constants, this is exactly what we expect to find for a primary operator of dimension $d=4$. In the case $N=2, k=1$ we identify this operator with $\left(u_{1}^{2}\right)_{2}$, or equivalently $\left[\left(u_{1}\right)_{1}\right]^{2}$, while in the case $N=1, k=2$ we identify this operator with $\left(u_{2}\right)_{1}$. As above, we can also describe descendants of this state, fill out the $\operatorname{Spin}(5)$ representations, etc.

\footnotetext{
${ }^{18} \mathrm{As}$ described in $\S 3.3$, the normalization we use for the Hamiltonian is also different in the two cases. In this section we will use the normalization of the $N=2, k=1$ case.
} 
Next, let us describe the 6 -form state $F_{6}$. After we blow up the $\mathbb{R}^{4} / \mathbb{Z}_{2}$ singularity, there is a non-trivial homology cycle corresponding to the blown up $\mathbf{C P}^{1}$, and a corresponding cohomology 2 -form. In this special case, we can choose a representative of the cohomology which is annihilated not only by $d$ but also by $d^{\dagger}=* d *$, restricted to the $v$ coordinates of the moduli space, and we will denote it by $\mathcal{F}_{2}=\left[\mathcal{F}_{2}(v)\right]_{i j} d v^{i} \wedge d v^{j}$. This form is concentrated near the blown-up singularity, and in the $\zeta \rightarrow 0$ limit gives a state that will be concentrated at the singularity. The 6 -form state that we find corresponds to $F_{6}=\delta^{4}(\tilde{x}) \prod_{i} d \tilde{x}^{i} \wedge \mathcal{F}_{2}$.

It is easy to compute the time evolution of this state, since $\mathcal{F}_{2}$ is annihilated by the Hamiltonian of the $v$ coordinates ${ }^{19}$. Thus, the evolution will be the same as for $N=1, k=1$, with a trivial evolution in the $v$ space. As before, this is consistent with this state having dimension 2, and we identify this state with $\left(u_{1}\right)_{2}$ for $N=2, k=1$ and with $\left(u_{1}\right)_{1}$ for $N=1, k=2$. The analysis of the descendants of this state is exactly as in the case of $N=1, k=1$ described above.

Note that in the computations described in this section, we found 2-point functions that did not depend on $\zeta$, and agreed exactly with our general predictions, described in $\S 5.2$. This depended on choosing particular representatives from each cohomology class. Generally, as described above, there will be $\zeta$-dependence and also different cohomology representatives will give different 2-point functions. But, the long time behavior will not depend on this.

\subsection{Comments on General $n$-point Functions}

In the previous sections we described the identification of some of the states in the quantum mechanics corresponding to the space-time theory for $P_{-}=$ $N / R$. These states are the momentum $N$ modes of some of the primary fields, $\phi\left(x^{+}, x^{i}, P_{-}=N / R\right)|0\rangle$. As described above, it is simple to compute 2-point functions of fields using these states ${ }^{20}$.

Obviously, we are also interested in computing higher $n$-point functions. We will briefly outline here a procedure for such a computation, although the details remain to be worked out [22]. In the space-time theory, we would like to compute an $n$-point function of the form

$$
\left\langle\phi_{1}\left(x_{1}, t_{1}\right) \phi_{2}\left(x_{2}, t_{2}\right) \cdots \phi_{n}\left(x_{n}, t_{n}\right)\right\rangle .
$$

\footnotetext{
${ }^{19}$ In the form language, $H \sim d d^{\dagger}+d^{\dagger} d$.

${ }^{20}$ In some cases the primary field we find is the non-singular part of a product of $l$ other primary fields, as detailed in $\S 6.2$, and then this 2-point function is also a special case of a $2 l$-point function, in which $l$ operators are at one point and $l$ at another.
} 
In DLCQ, the object that we may try and calculate is of the form

$$
\left\langle\phi_{1}\left(x_{1}^{i}, x_{1}^{+}, P_{-, 1}=\frac{k_{1}}{R}\right) \cdots \phi_{n}\left(x_{n}^{i}, x_{n}^{+}, P_{-, n}=\frac{k_{n}}{R}\right)\right\rangle .
$$

To simplify the discussion we will limit ourselves to correlation functions in which the operators with positive $P_{-}$appear at smaller values of $x^{+}$than the operators with negative $P_{-}$. The prototypical problem is then to construct a state of the form

$$
\phi_{1}\left(x_{1}^{i}, x_{1}^{+}, P_{-, 1}=\frac{k_{1}}{R}\right) \phi_{2}\left(x_{2}^{i}, x_{2}^{+}, P_{-, 2}=\frac{k_{2}}{R}\right)|0\rangle,
$$

with $k_{1}, k_{2}>0$, which can then be used to compute 3 -point or 4 -point functions ${ }^{21}$.

The state (45) has longitudinal momentum $P_{-}=\left(k_{1}+k_{2}\right) / R$, so it should appear in the quantum mechanics in the $N$-instanton moduli space (where $\left.N=k_{1}+k_{2}\right)$. Without loss of generality we can assume that $x_{2}^{+}<x_{1}^{+}$. We start by constructing the state $\phi_{2}\left(x_{2}^{i}, x_{2}^{+}, P_{-, 2}=k_{2} / R\right)|0\rangle$. This is a state in the quantum mechanics on the moduli space of $k_{2}$ instantons. At DLCQ time $x_{2}^{+}$it is localized (as described above) at the origin of the Higgs branch, and at some point $x_{2}^{i}$ in the center of mass coordinates. We can propagate this state in the quantum mechanics (using the Hamiltonian) up to the time $x_{1}^{+}$. Generally the state will spread on the Higgs branch. Next, we would like to transfer this state to the quantum mechanics on the $N$ instanton moduli space, and to add the operator $\phi_{1}$.

This can be done in the following way. Within the $k_{1}+k_{2}$ instanton moduli space we can focus on a submanifold $\mathcal{W}$ where $k_{1}$ of the instantons have shrunk to zero size at some point $x_{1}^{i}$. The submanifold $\mathcal{W}$ is roughly the moduli space of $k_{2}$ instantons. These are the remaining instantons which can be in an arbitrary instanton configuration. The space transverse to $\mathcal{W}$ is (locally) roughly the moduli space of $k_{1}$ instantons, as going away from this space corresponds to the $k_{1}$ instantons growing to a finite size (or moving around). On $\mathcal{W}$ we can put the wave function that we obtained above from the propagation of the $\phi_{2}$ state on the $k_{2}$ instanton moduli space. We can multiply this by a wave function on the transverse space which is the wave function that corresponds to the operator $\phi_{1}$. Since $\phi_{1}$ is a local operator, this wave function is also localized on $\mathcal{W}$ (which is the origin of the $k_{1}$ instanton moduli space). The resulting state corresponds to (45) at time $x_{1}^{+}$.

Once we have such a state we can propagate it in time, and then either calculate its overlap with some state in the $N$ instanton moduli space to

\footnotetext{
${ }^{21}$ Note that this state generally includes operators at different "DLCQ time" $x^{+}$. This should be understood as involving a time evolution of these states (using the Hamiltonian) to the same $x^{+}$.
} 
obtain, say, a 3-point function, or we can again embed it into an instanton moduli space of higher instanton number and calculate a higher $n$-point function.

\section{Acknowledgments}

We would like to thank J. Distler, R. Entin, N. Hitchin, S. Kachru, S. Minwalla, G. Moore, J. Morgan, H. Nakajima, Y. Oz, E. Silverstein and E. Witten for useful discussions. We are particularly indebted to T. Banks and L. Susskind for collaboration in the early stages of this work. OA was supported in part by DOE grant \#DE-FG02-96ER40559. The work of MB was supported by NSF grant NSF PHY-9512835. The work of NS was supported by DOE grant \#DE-FG02-90ER40542.

\section{References}

[1] T. Banks, W. Fischler, S. Shenker, and L. Susskind, Phys. Rev. D55 (1997) 112.

[2] T. Maskawa and K. Yamawaki, Prog. Theor. Phys. 56 (1976) 270.

[3] A. Casher, Phys. Rev. D14 (1976) 452.

[4] C. B. Thorn, Phys. Rev. D19 (1979) 639; C. B. Thorn, Phys. Rev. D20 (1979) 1435; C. B. Thorn, Phys. Rev. D20 (1979) 1934.

[5] H. C. Pauli and S. J. Brodsky, Phys. Rev. D32 (1985) 1993; H. C. Pauli and S. J. Brodsky, Phys. Rev. D32 (1985) 2001.

[6] L. Susskind, "Another Conjecture About M(atrix) Theory", hepth/9704080.

[7] N. Seiberg, Phys. Rev. Lett. 79 (1997) 3577.

[8] M. R. Douglas, H. Ooguri and S. H. Shenker, Phys. Lett. 402B (1997) 36; M. Dine and A. Rajaraman, "Multigraviton Scattering in the Matrix Model", hep-th/9710174; M. R. Douglas and H. Ooguri, "Why Matrix Theory is Hard", hep-th/9710178; D. Kabat and W. Taylor, "Spherical Membranes in Matrix Theory", hep-th/9711078.

[9] S. Hellerman and J. Polchinski, "Compactification in the Lightlike Limit", hep-th/9711037.

[10] T. Banks, "Matrix Theory", hep-th/9710231. 
[11] S. J. Brodsky, H. Pauli and S. S. Pinsky, "Quantum Chromodynamics and Other Field Theories on the Light Cone", hep-ph/9705477, submitted to Physics Reports.

[12] D. Bigatti and L. Susskind, "A Note on Discrete Light Cone Quantization", hep-th/9711063.

[13] E. Witten, "Some Comments on String Dynamics", contributed to STRINGS'95: "Future Perspectives in String Theory", Los Angeles, CA, 13-18 March 1995.

[14] A. Strominger, Phys. Lett. 383B (1996) 44.

[15] O. Aharony, M. Berkooz, S. Kachru, N. Seiberg, and E. Silverstein, Adv. Theor. Math. Phys. 1 (1998) 148.

[16] E. Witten, "On the Conformal Theory of the Higgs Branch", hepth/9707093.

[17] D. A. Lowe, " $E_{8} \times E_{8}$ Small Instantons in Matrix Theory", hepth/9709015.

[18] O. Aharony, M. Berkooz, S. Kachru, and E. Silverstein, "Matrix Description of $(1,0)$ Theories in Six Dimensions", hep-th/9709118.

[19] M. Rozali, Phys. Lett. 400B (1997) 260; M. Berkooz, M. Rozali and N. Seiberg, Phys. Lett. 408B (1997) 105.

[20] E. Witten, Nucl. Phys. B507 (1997) 658.

[21] J. Maldacena, "The Large $N$ Limit of Superconformal Field Theories and Supergravity", hep-th/9711200.

[22] O. Aharony, M. Berkooz and N. Seiberg, work in progress.

[23] J. Brodie and S. Ramgoolam, "On Matrix Models of M5-Branes", hepth/9711001.

[24] P. Claus, R. Kallosh and A. Van Proeyen, "M 5-brane and Superconformal $(0,2)$ Tensor Multiplet in 6 Dimensions", hep-th/9711161.

[25] O. J. Ganor and S. Sethi, "New Perspectives on Yang-Mills Theories with Sixteen Supersymmetries", hep-th/9712071.

[26] E. Witten, Nucl. Phys. B443 (1995) 85.

[27] M. Berkooz and M. Douglas, Phys. Lett. 395B (1997) 196.

[28] M. R. Douglas, "Branes Within Branes", hep-th/9512077. 
[29] M. Atiyah, V. Drinfeld, N. Hitchin, and Y. Manin, Phys. Lett. 65B (1978) 185.

[30] W. Nahm, Nucl. Phys. B135 (1978) 149.

[31] L. Susskind, unpublished.

[32] C. Vafa and E. Witten, Nucl. Phys. B431 (1994) 3.

[33] H. Nakajima, "Resolutions of Moduli Spaces of Ideal Instantons on $\mathbb{R}^{4}$ ", in "Topology, Geometry and Field Theory", Fukaya, Furuta, Kohno and Kotschick, eds., World Scientific.

[34] S. Sethi and M. Stern, "D-Brane Bound States Redux", hep-th/9705046; P. Yi, Nucl. Phys. B505 (1997) 307; M. Porrati and A. Rozenberg, "Bound States at Threshold in Supersymmetric Quantum Mechanics", hep-th/9708119, version to appear in Nucl. Phys. B.

[35] P. K. Townsend, Phys. Lett. 373B (1996) 68.

[36] A. Connes, M. R. Douglas and A. Schwarz, "Noncommutative Geometry and Matrix Theory: Compactification on Tori", hep-th/9711162.

[37] N. A. Obers, B. Pioline and E. Rabinovici, "M-Theory and U-Duality on $T^{d}$ with Gauge Backgrounds", hep-th/9712084.

[38] B. de Wit, K. Peeters and J. C. Plefka, "Supermembranes and Supermatrix Models", hep-th/9712082.

[39] O. Aharony, Nucl. Phys. B476 (1996) 470; E. Bergshoeff, M. de Roo and T. Ortin, Phys. Lett. 386B (1996) 85; P. Pasti, D. Sorokin and M. Tonin, Phys. Lett. 398B (1997) 41; I. Bandos, K. Lechner, A. Nurmagambetov, P. Pasti, D. Sorokin and M. Tonin, Phys. Rev. Lett. 78 (1997) 4332; M. Aganagic, J. Park, C. Popescu and J. H. Schwarz, Nucl. Phys. B496 (1997) 191; I. Bandos, N. Berkovits and D. Sorokin, "Duality-Symmetric Eleven-Dimensional Supergravity and its Coupling to M-branes", hep-th/9711055.

[40] E. Silverstein and E. Witten, Nucl. Phys. B444 (1995) 161.

[41] E. Witten, Nucl. Phys. B202 (1982) 253; D. Friedan and P. Windey, Nucl. Phys. B235 (1984) 395; L. Alvarez-Gaume, Comm. Math. Phys. 90 (1983) 161.

[42] S. Minwalla, "Restrictions Imposed by Superconformal Invariance on Quantum Field Theories", hep-th/9712074. 
[43] J. Distler, private communication.

[44] K. Yoshioka, J. Reine Angew. Math. 453 (1994) 193, Proposition 5.4.

[45] I. Grojnowski, H. Nakajima and K. Yoshioka, in preparation. 